\title{
1 Benefits and Challenges of Using Virtual Primary Care During the COVID-19 2 Pandemic: From Key Lessons to a Framework for Implementation
}

3

27 Corresponding author

(

Name: Ana Luisa Neves W2 1NY

Telephone: 02033127259

Fax number: 02033126309

Word count: 3,970 words
Authors: Edmond Li, Rosy Tsopra, Geronimo Larrain Gimenez, Alice Serafini, Gustavo Gusso, Heidrun Lingner, Maria Jose Fernandez, Greg Irving, Davorina Petek, Robert Hoffman, Vanja Lazic, Memarian Ensieh, Tuomas Koskela, Claire Collins, Sandra Milena Espitia, Ana Clavería, Katarzyna Nessler, Braden Gregory O’Neill, Kyle Hoedebecke, Mehmet Ungan, Liliana Laranjo, Saira Ghafur, Gianluca Fontana, Azeem Majeed, Josip Car, Ara Darzi, Ana Luisa Neves

Full address of the authors: Institute of Global Health Innovation, Room 1035/7, QEQM Wing, St Mary's Campus, London W2 1NY

Target journal: BMJ Quality and Safety

Address: Institute of Global Health Innovation, Room 1035/7, QEQM Wing, St Mary's Campus, London

E-mail: ana.luisa.neves14@ic.ac.uk 


\section{Abstract}

Background: With the onset of COVID-19, general practitioners (GPs) and patients worldwide swiftly transitioned from face-to-face to digital remote consultations. There is a need to evaluate how this global shift has impacted patient care, healthcare providers, patient and carer experience, and health systems.

Objective: We explored GPs' perspectives on the main benefits and challenges of using digital remote care.

41 Methods: GPs across 20 countries completed an online questionnaire between June - September 2020.

42 GPs' perceptions on main barriers and challenges were explored using free-text questions. Thematic

43 analysis was used to analyse the data.

44 Results: 1,605 respondents participated in our survey. The benefits identified included reducing COVID-

4519 transmission risks, guaranteeing access and continuity of care, improved efficiency, faster access to care,

46 improved convenience and communication with patients, greater work flexibility for providers, and

47 hastening the digital transformation of primary care and the accompanying legal frameworks.

48 Main challenges included patient's preference for face-to-face consultations, digital exclusion, lack of physical examinations, clinical uncertainty, delays in diagnosis and treatment, overuse and misuse of digital remote care, and unsuitability for certain types of consultations. Other challenges include the lack of formal guidance, higher workloads, remuneration issues, organisational culture, technical difficulties,

52 implementation and financial issues, and regulatory weaknesses.

53 Conclusion: At the frontline of care delivery, GPs can provide important insights on what worked well, 54 why, and how. Lessons learned during the emergency phase can be used to inform the stable adoption of 55 virtual care solutions, and co-design processes and platforms that are technologically robust, secure, and 56 supported by a strategic long-term plan.

57

Keywords: Telemedicine, primary care, quality of care, patient safety, digital health 


\section{Introduction}

For decades, there have been many initiatives to implement virtual care into healthcare systems. In the US, Kaiser Permanente offers secure email communication and routine telephone and video consultations [1]. In the UK, the use of telephone consultations is commonplace [2]. Many other healthcare systems worldwide have advocated similarly for a virtual approach [3-9].

The use of virtual care, either via telephone, video, or online technologies, has potential implications on the six domains of quality of care: timeliness, efficiency, patient-centredness, effectiveness, safety, and equity [10]. Virtual consultations can reduce delays in the diagnosis and treatment, thus improving timeliness [11]. They can also facilitate access for patients living in isolated areas, and reduce inequities in care delivery [12-14]. Remote care can improve primary care efficiency by acting as a gatekeeper by remotely triaging patients, identifying those who require urgent face-to-face care from those who can be managed virtually [15-17]. While some studies have suggested that virtual care can improve efficiency and generate time savings [18,19], others did not find a statistically significant reduction [20]. Remote care can support the delivery of more patient-centred care and the development of self-management skills [21,22]. Virtual care can be effective in the management of chronic conditions, including chronic obstructive pulmonary disease, heart failure, and diabetes mellitus [23]. Studies have also suggested improvements on patient safety and a potential reduction in hospital admissions [20,23]. However, despite the promised benefits, virtual care has been integrated slowly into primary care. A wide range of obstacles have limited widespread adoption, including cultural, regulatory and policy, industrial and technical, knowledge, financial, and market-related barriers [24].

With the onset of COVID-19, the primary care landscape was radically transformed [25,26]. Many countries have released national guidance encouraging the use of virtual triage and consultation systems [26]. In a few weeks, General Practitioners (GPs) and patients worldwide transitioned from face-to-face consultations to virtual care [27]. Whilst previous evidence surrounding the use of remote care in general practice came from relatively small and local clinical trials, this pandemic forced patients, healthcare providers, and healthcare systems to embrace virtual consultations as the primary route to access care. Thus, this presents us with a unique opportunity to learn more from this global real-life experiment, identify the main challenges and benefits experienced, and incorporate these lessons into the future of virtual primary care [28].

As healthcare providers on the frontline of care delivery, GPs are excellently placed to identify the benefits and challenges of using digital tools for remote care. This study aimed to explore GPs' perspectives on the main benefits and challenges of using virtual care, mapping them against the main domains of quality of care whenever possible. As a secondary aim, we sought to summarise findings as a framework of recommendations for the implementation of virtual care delivery in primary care settings.

\section{Methods}

96 The study used an online questionnaire survey of GPs in twenty countries. Recruitment took place between June - September 2020.

\section{Study population}

100

The inSIGHT Research Group is a worldwide collaboration of primary care researchers exploring the impact of the COVID-19 pandemic on the adoption of virtual primary care. The research group is spread across 20 countries (Australia, Brazil, Canada, Chile, Colombia, Croatia, Finland, France, Germany, Ireland, Israel, Italy, Poland, Portugal, Slovenia, Spain, Sweden, Turkey, United Kingdom, United 
medRxiv preprint doi: https://doi.org/10.1101/2021.07.28.21261021; this version posted July 29, 2021. The copyright holder for this preprint (which was not certified by peer review) is the author/funder, who has granted medRxiv a license to display the preprint in perpetuity.

It is made available under a CC-BY 4.0 International license.

States). Participants were eligible for the survey if they were GPs working in these countries between March and September 2020.

\section{Sampling}

Each local lead sent an email invitation to GPs in their country and shared the link to the survey in social media channels (e.g., LinkedIn, Twitter, Facebook). Local leads who had difficulty achieving the minimum number required used snowballing to increase the number of responses. Snowballing is a recognised technique for recruiting hard-to-reach populations in health studies [29-31].

\section{Description of the questionnaire}

The questionnaire included 30 items assessing GPs' perspectives on the adoption and experience of virtual care solutions during the COVID-19 outbreak. Participants' characteristics were collected, including age, gender, country, practice setting, number of years of experience as GP, and involvement in teaching activities. GPs' perceptions on the main benefits and challenges of using virtual care were assessed using free-text questions.

\section{Data analysis}

Participants' characteristics were analysed using descriptive statistics. Two independent researchers systematically reviewed the transcripts using the framework analysis method, which includes five main stages: familiarisation, identifying a thematic framework, indexing, charting, and mapping and interpretation [19]. At every stage of the data analysis process, the coding framework was kept deductive and inductive, allowing the inclusion of emergent themes. The coding tree was shared between all researchers for iterative refinement until consensus was reached. Resultant themes, subthemes, and the relationships between them, will be visualised using the Miro online whiteboard application [32]. As participants did not provide consent for further contact, it was not possible to ask them to provide feedback on the findings. The Consolidated Criteria for Reporting Qualitative Studies checklist was used to ensure the study meets the recommended standards of qualitative data reporting.

\section{Ethics}

Overall ethical approval for this project was granted by the Imperial College Research Ethics Committee (ICREC) (Reference 20IC5956). This is a dedicated ethics oversight body at Imperial College London for all health-related research involving human participants. In addition, whenever necessary, local ethical approval and relevant permissions in the respective participating countries were also obtained.

\section{Results}

\section{Participants' characteristics}

1,605 participants participated in the questionnaire. Most respondents (79.3\%) were aged 30-59 years, and $60.9 \%$ were female $(n=978)$. Most of the participants have been working as GPs for a minimum of 5 years $(79.1 \%, \mathrm{n}=1,329)$ and reported being involved in teaching activities $(63.7 \%, \mathrm{n}=1,023)$. More than half $(62.5 \%)$ worked in an urban setting $(\mathrm{n}=1,004)$. A full description of the participants, including a breakdown per country, is shown in Table 1. 
medRxiv preprint doi: https://doi.org/10.1101/2021.07.28.21261021; this version posted July 29, 2021. The copyright holder for this preprint (which was not certified by peer review) is the author/funder, who has granted medRxiv a license to display the preprint in perpetuity.

\section{It is made available under a CC-BY 4.0 International license.}

Table 1. Participants' characteristics

\begin{tabular}{|c|c|}
\hline Characteristics & $n(\%)$ \\
\hline $\begin{array}{l}\text { Gender, } \boldsymbol{n}(\%) \\
\text { Female } \\
\text { Male } \\
\text { No response } \\
\text { Other }\end{array}$ & $\begin{array}{c}978(60.9) \\
616(38.4) \\
10(0.6) \\
2(0.1)\end{array}$ \\
\hline $\begin{array}{l}\text { Age category, } \boldsymbol{n}(\%) \\
\text { Under } 30 \\
30-39 \\
40-49 \\
50-59 \\
60-69 \\
70+ \\
\text { Prefer not to answer }\end{array}$ & $\begin{array}{c}101(6.3) \\
531(33.1) \\
415(25.8) \\
327(20.4) \\
210(13.1) \\
18(1.1) \\
4(0.2)\end{array}$ \\
\hline $\begin{array}{l}\text { Country, } \boldsymbol{n}(\%) \\
\text { Australia } \\
\text { Brazil } \\
\text { Canada } \\
\text { Chile } \\
\text { Colombia } \\
\text { Croatia } \\
\text { Finland } \\
\text { France } \\
\text { Germany } \\
\text { Ireland } \\
\text { Israel } \\
\text { Italy } \\
\text { Poland } \\
\text { Portugal } \\
\text { Slovenia } \\
\text { Spain } \\
\text { Sweden } \\
\text { Turkey } \\
\text { UK } \\
\text { United States of America }\end{array}$ & $\begin{array}{c}99(6.2) \\
53(3.3) \\
53(3.3) \\
58(3.6) \\
63(3.9) \\
62(3.9) \\
54(3.4) \\
62(3.9) \\
50(3.1) \\
267(16.6) \\
79(4.9) \\
97(6) \\
66(4.1) \\
95(5.9) \\
77(4.8) \\
100(6.2) \\
76(4.7) \\
63(3.9) \\
77(4.8) \\
54(3.4)\end{array}$ \\
\hline $\begin{array}{l}\text { Setting, } \boldsymbol{n}(\%) \\
\text { Mixed } \\
\text { Rural } \\
\text { Urban }\end{array}$ & $\begin{array}{l}358(22.3) \\
244(15.2) \\
1004(62.5)\end{array}$ \\
\hline $\begin{array}{l}\text { Experience, } \boldsymbol{n}(\%) \\
<5 \text { years } \\
5-10 \text { years } \\
10-15 \text { years } \\
15-20 \text { years } \\
>20 \text { years }\end{array}$ & $\begin{array}{l}336(20.9) \\
359(22.4) \\
242(15.1) \\
174(10.8) \\
495(30.8)\end{array}$ \\
\hline $\begin{array}{l}\text { Teaching activities, } \boldsymbol{n}(\%) \\
\text { Yes } \\
\text { No } \\
\text { Prefer not to answer }\end{array}$ & $\begin{array}{c}1023(63.7) \\
569(35.4) \\
14(0.9)\end{array}$ \\
\hline
\end{tabular}




\section{Main benefits}

Benefits clustered around three main themes (Figure 1, Textbox 1): benefits for quality and safety of care (i.e., safety, effectiveness, equity, efficiency, timeliness, patient-centredness), for health care professionals, and for health care systems.

Benefits for quality of care

The reduced risk of COVID-19 transmission was identified as the main safety benefit. Participants also recognised that virtual care had benefits on effectiveness, ensuring accessibility and continuity of care for both COVID-19 and non-COVID-19 patients. Participants highlighted that virtual care has improved equity in access to care for some groups of patients (e.g., frail elderly people, those with mobility issues, or living far from clinics or in geographically isolated areas). Improvements concerning the efficiency of care included the ability to perform remote triage, reduce unnecessary face-to-face visits (i.e., mild illnesses, prescription renewal, or administrative tasks), and optimise the use of human resources (i.e., enhancing communication between providers). Participants also believed that virtual care improved timeliness, including less time spent in physical dislocation, waiting for administrative procedures, or for clinical appointments. Participants described several benefits for patient-centredness, such as improved convenience and communication, and a positive effect on patient-doctor relationship - often against their prior expectations. GPs acknowledged the importance of virtual care on patient empowerment, by

Textbox 1. Main benefits: thematic analysis of the participants' narratives (Table is author's original work)

\section{THEME 1: BENEFITS FOR QUALITY OF CARE}

\section{Subtheme 1.1. Improved safety: Reduced risk of COVID-19 transmission}

- "[Digital technologies] allowed decreasing the exposure to infection to both health providers as well as patients" (ID 312, Colombia)

Subtheme 1.2. Improved effectiveness: (access and continuity of care) and equity

- "Able to rapidly adapt to the new reality of Covid. We closed the front door but could still keep providing patient care." (ID 924, Ireland)

- "Continuing care with patients who otherwise might not have attended the practice because of concern about infection." (ID 1570, Australia)

- "the possibility of taking care of the patients even without seeing them.. especially [ensuring the] patient did not feel abandoned." (ID 215, Italy)

- " "Digital remote care] allowed us to remain accessible and in contact with our patients (ID253, Spain)

- "Continuity of care for patients with chronic diseases; psychological support or therapy for patients with mental health conditions remotely." (ID 347, France)

- "Facilitated the follow-up of diagnosed or suspected patients, favouring that isolation does not imply loss of care and detection of complications. Patients and their families or caregivers were supported" (ID387, Spain)

- "Able to continue to care for patients, especially those with acute needs or fragile chronic needs." (ID 566, USA)

- " "[we were] able to provide care for people who have various barriers to accessing in-person care (frail elderly; people who cannot get time off work; people with physical disabilities)" (ID 1446, Canada)

- "Helpful for patients living far from the practice, with infectious diseases, disabilities. Helpful for follow up visits." (ID 827, Poland)

- "The ability to provide care in remote communities that otherwise have to family physician and would traditionally resort to presenting to the ER" (ID 1336, Canada) 
Subtheme 1.3. Improved efficiency: triage and practice management

- "Ability to triage and bring in patients who need F2F: (408, UK)

- "Allow qualified triage by doctors, giving access to face-to-face to the patients that do need it (ID 126, Portugal)

- "By doing a prior telephone screening, face-to-face assistance and follow-up could be better prioritized. Before, almost all patients requested a face-to-face appointment as soon as possible without any prioritization or filtering criteria. That was a great improvement." ((ID401, Spain)

- "Help filter patients and problems, so that only cases that are critical and need a prompt resolution make it to the healthcare centres" (ID 274, Chile)

- "Optimise health resources, especially human resources" (ID 870, Chile)

- 'Optimisation of resources to answer the healthcare needs of the population' (ID 327, Portugal)

- 'We have learned that many issues can be taken care of without a face-to-face consultation. This seems to be efficient for both the doctors and the patients' (ID 10, Finland)

Subtheme 1.4. Improved timeliness

- "We had faster access to consultation with colleagues in hospitals for chronic patients, via e-referral without the colleague seeing the patient live." (ID 1075, Croatia)

- "Shorter waiting times to receive care" (ID 149, Colombia)

- Meetings with others are efficient, timely and seem easier. No time wasted with travel etc (ID 833, $U K \& N I)$

- "Possibility of accessing consultations in a more expedited way" (ID 436, Chile)

- Ability to deliver care in a timely fashion. I was pleasantly surprised that the majority of visits were easily done on the telephone or via videoconference without compromising patient safety or satisfaction." (ID 409, Canada)

Subtheme 1.5. Improved patient-centredness: convenience, communication, and patient empowerment

- "The biggest benefits have been increasing access to care and for allowing visits to be able to more seamlessly fit into patients' lives." (ID 1282, USA)

- "Reduce patient discomfort for access to care" (246, Italy)

- "[Digital technologies] strengthened communication with patients to offer help and support" (ID 1467, Colombia)

- "With technological support [..] the relationship is integrated and modified, often enriched; this actually goes against the clichés of depersonalisation of relationships very often reported by professionals of my generation" (ID 223, Italy)

- "[Digital remote care] allowed us to (...) delegate greater responsibilities in self-management of minor health problems (ID253, Spain)

- “(...) allowed us to remain accessible and in contact with our patients, and delegate greater responsibilities in self-management of minor health problems (ID253, Spain)

- "Educating patients, self-managing minor health issues and hopefully better communication" (ID 1085, Croatia)

\section{THEME 2: BENEFIT FOR HEALTH CARE PROFESSIONALS}

\section{Subtheme 2.1. Remote working}

- 'Possibility to work even if I was in quarantine' (ID 124, France)

- 'Gave me the ability to work from home while I suffered from mild COVID.' (ID 1516, Sweden)

- "The possibility of working remotely allowed me to preventing burnout and conciliating family and professional demands" (ID 47, Portugal)

- "Telephone consultations normalized and kept people out of office who needed to be home - allowed clinicians to be away from office when sick and still provide care" (ID 1252, Canada)

Subtheme 2.2. More control over schedule

- "Better management of appointments by the physician" (ID 55, Portugal)

- "More control for me over my schedule" (ID 110, Israel) 


\section{THEME 3: BENEFITS FOR HEALTH CARE SYSTEMS}

\section{Subtheme 3.1. Hastening the digital transformation}

- "Working through video consultations was a very positive experience that I never would have tried without the pandemic.' (ID 809, Sweden)

- "[It was an] eye opening experience for both doctors and patients, [showing] that telemedicine is helpful and can be safely used.' (ID 827, Poland)

- "It was a chance to experience the digital consultation and to see that primary health care can use more advanced digital solutions for taking care of patients. (ID 951, Turkey)

- "Learning that one can treat patients in many cases by their symptoms only without a frontal clinical exam" (34, Israel)

- "I realised that a lot of things can be done without face-to-face contact which leaves me more time for patients who need to be examined" (Slovenia, ID 838)

- "Realize that clinical and non-clinical activities can be performed at a distance saving time, transportation and energy, which could be maintained after the pandemic" (ID 193, Chile)

- "Active and transformative learning that a significant proportion of health concerns can be managed remotely (ID 40, Portugal)

- "It increased the digital skills of doctors and patients" (295, Italy)

- "Upgrade of electronic connection/data transfer between health fond, computer/program provider and family doctor" (ID 1109, Croatia)

Subtheme 3.2. Changing legal and regulatory frameworks

- "We started online consultation in primary care which was prohibited in Poland before" (ID 556, Poland)

- "In Brazil, the biggest benefit was a political one, since only now teleconsultations were made legal in the country." (ID 79, Brazil)

- "I actually got paid for some of the stuff we normally have to do in lunch break or after hours for free.". (ID 1560, Australia)

- [Receiving] payments for such services" (ID 587, USA)

170

171

172

173

174

175

176

177

178

179

180

181

182

183

Benefits for healthcare professionals

Some respondents identified the ability to work remotely as a major benefit, as well as having more control over their schedule.

Benefits for healthcare systems

Many respondents underscored the use of virtual care gained during the COVID-19 pandemic has been a major factor hastening the digital transformation (i.e., increasing awareness and trust, improving digital skills of both patients and providers, and upgrading technical capacity). Some participants mention the quick deployment of new digital opportunities (e.g., access to e-referrals, e-prescriptions, and electronic processing of fit-to-work certificates). Other benefits included changes in legal and regulatory frameworks, particularly in what concerns the legal context and remunerations of remote care tasks. 


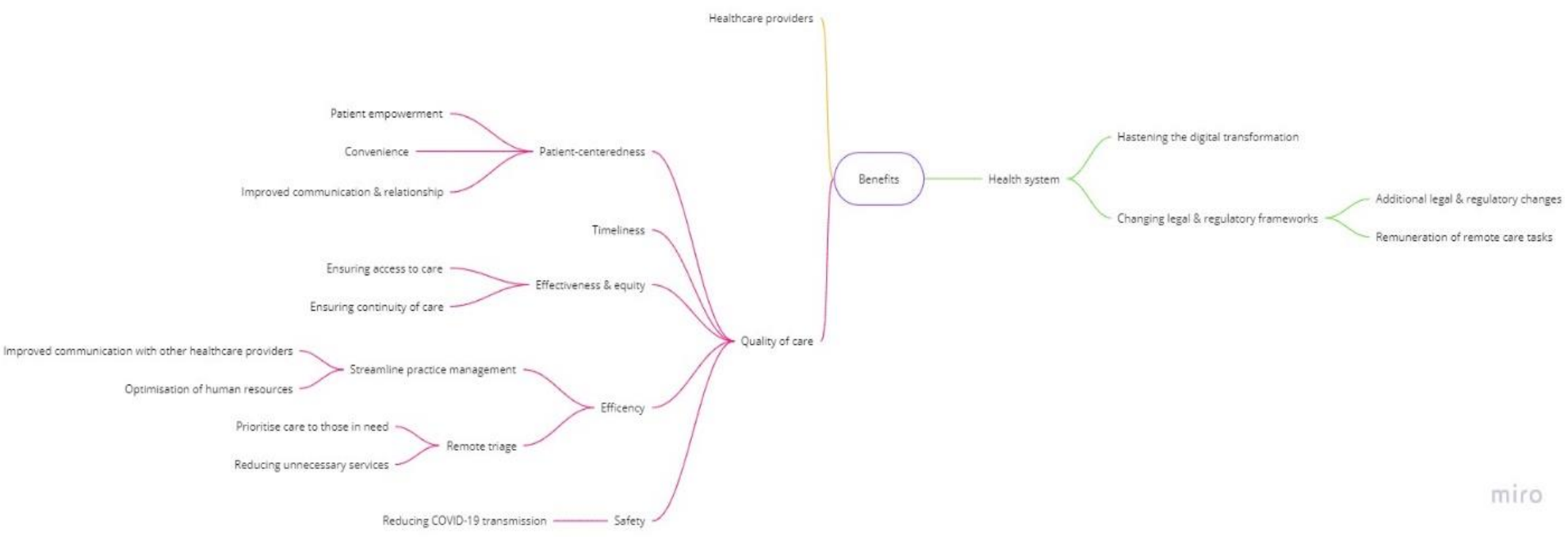

185

\section{Main challenges}

Challenges were broadly summarised into three main areas (Figure 2, Textbox 2): for quality of care, for healthcare providers, and for health care systems.

\section{Quality of care}

Participants were concerned that remote care can negatively impact some aspects of patient-centredness, including patient preferences and the patient-doctor relationship. In fact, one of the identified detractors to the use of virtual care was patients' preference for traditional face-to-face consultations. GPs also reported that it was often difficult to gauge a patient's body language and emotions through digital video/audio channels, thus impairing their ability to build rapport, express empathy, and provide more holistic care to their patients. GPs acknowledge this to have a potential negative impact on patients' wellbeing, since physically attending appointments was an opportunity for social interaction for socially isolated individuals. For clinicians, remote consultations posed additional challenges for patient communication, particularly in emotionally difficult situations, or when there was a need to ensure that their medical advice was properly understood.

Participants expressed concerns regarding a negative impact on equity, particularly regarding the digital exclusion of vulnerable patients. They noted that many patients lack access to reliable internet service, and computers or smartphones to conduct video consultations. Even in circumstances where the hardware is present, some patients may lack the digital literacy and skills needed to independently set up and use relevant digital software.

The most prominent safety challenge was the inability to perform clinical examinations and assess physical signs to inform clinical decision-making. While some of these issues could be mitigated through careful history taking and patient self-reporting (e.g., photographs of visible lesions and remote monitoring devices, such as blood pressure monitors, glucometers, oximeters), GPs mention that few patients had the necessary devices, and if they had, would often struggle to use them and report the results back. Dealing with uncertainty, a recognised challenge in primary care, seems to be aggravated by these factors and contribute to an increased fear of misdiagnosis and inappropriate clinical management. 
GPs openly expressing concerns that digital remote care could delay diagnosis and treatment. Respondents reported that these delays could be caused by patients' reluctance to use virtual care, lack of access and digital skills to use these tools, and communication challenges (i.e., to effectively convey non-verbal clinical information).

GPs also emphasised some potential threats to the efficiency of care delivery. While some patients expressed hesitancy to use virtual care, others were overly enthusiastic about having a more direct line of communication with their healthcare provider, often resulting in misuse and overuse. This increase in patient demand translated into an increase in GPs' workload. Finally, a few quotes highlighted that virtual care may not be an effective solution for certain types of consultations.

Textbox 2. Main challenges: thematic analysis of the participants' narratives (Table is author's original work)

\section{THEME 1: CHALLENGED FOR QUALITY OF CARE}

Subtheme 1.1. Challenges for patient-centredness

- "Patient preference for face to face due to issues such as poor hearing on devices and patients not having ability to use technology." (ID 1561, Australia)

- "Patients and doctors prefer face to face, many patients pay in Ireland, online isn't acceptable [to them]" (ID 1019, Ireland)

- "Patients are accustomed to meet in GP clinics to talk with one another, especially older patients, lonely, they are waiting for meetings with doctors frequently too. Some have problems with electronic devices." (ID 556, Poland)

- "Lack of human contact, facial and body language. Difficult to show emotions and compassion. I used to close my eyes and imagine the patient on the other side of my table. I expend more time searching the suitable words to overcome the lack of body language" (ID 62, Portugal)

- "Difficulty to build rapport with patients who you don't already know - less trust of HCP" (ID 1033, UK\&NI)

- "Harder for people who are socially isolated, coming to the clinic was an important human interaction, form of meeting other patients, the extended team etc." (ID 630, Canada)

\section{Subtheme 1.2. Challenges for equity, and digital exclusion}

- "Some elderly (the ones with the biggest need) have problems using a telephone, let alone a computer..." (ID 1518, Sweden)

- "Unfortunately, a lot of elderly patients (...) do not know how to use digital technology (email, messenger, etc) and in my opinion were deprived during the pandemic." (ID 1075, Croatia)

- "[an unintended consequence was] Neglecting the less technology-oriented patients, which are also the most vulnerable ones - the elderly, the underprivileged, the immigrants etc, and in fact giving preference to the younger healthier patients, thereby deepening health inequality" (ID 254, Israel)

\section{Subtheme 1.3. Challenges for safety}

- "[It is] much harder to make decisions on the phone without examining the patient. Often patients refused to come in for appointments and didn't understand the lack of ability to assess a certain presenting complaint over the phone (e.g., abdominal pain).” (ID 1598, Australia)

- "Not all necessary information concerning a patient's condition can be transmitted digitally/by phone and this causes a risk of not noticing a critical symptom/change in a patient's condition" (ID 8, Finland)

- "I found that I made more mistakes when making the initial diagnosis over email / our app / phone. If there is only a one-way written communication, then it is more easy to get seduced by the patient's view and not valorise objective facts, as we would in a face-to-face consultation." (ID 1320, Croatia) 


\section{Subtheme 1.4. Challenges for timeliness}

- "[A potential challenge was] not detecting patients with for instance atrial flutter because not seeing and examining them. More patients with symptoms of serious diseases are reluctant to seek care. Some are probably missed and come when the disease has progressed. I tried to be aware of this and arrange secure ways to investigate the patients." (ID 317, Sweden)

Subtheme 1.5. Challenges for effectiveness

- "Telephone consultations instead of face-to-face are less effective in many cases. It works fine for minor problems" (ID 440, Sweden)

- "Harder to check up on chronic diseases patients (prescribing medications without physical examination), easier to miss potentially dangerous symptoms, harder to counsel patients in psychological distress (video calls are not common)" (ID 499, Poland)

- "Online tools that don't work as well as they should (for example don't provide all the necessary information for a professional or lead to another face-to-face consultation anyway)" (ID 18, Finland)

\section{Subtheme 1.6. Challenges for efficiency}

- "Certain patients have been calling in much more frequently for minor issues that they would normally not have contacted their primary care provider for." (ID 409, Canada)

- "Make people understand what the priorities are and the appropriate times. I have kept the phone on for emergencies and have sometimes been contacted for irrelevant things at inconvenient times." (ID 169, Italy)

- "Too much access for system abusive patients" (ID 42, Portugal)

\section{THEME 2: CHALLENGES FOR HEALTHCARE PROFESSIONALS}

\section{Subtheme 2.1. Lack of guidance and support}

- "[One challenge was] the lack of previous training neither during undergraduate nor [during] postgraduate training. I have taken some online training. Also, the challenge was the lack of guidelines for primary care doctors." (ID 158, Poland)

\section{Subtheme 2.2. Higher workload and burnout}

- "It takes more time to do a remote consultation. It requires preparing the call, reviewing the clinic history and having a checklist according to the patient's category. If a face-to-face consultation takes $20 \mathrm{~min}$, a remote consultation requires 40-50 min average" (ID 244, Colombia)

- "I felt that my resources were drained by the phone communication. I am less concentrated, more nervous and less compassionate about patients" (ID 286, Israel)

- "Found myself working longer hours as working from home made it much harder to set boundaries around when I stopped work. (ID 1590, Australia)

- "I would contend that the majority of GPs would prefer to consult with their patients' in the real world more often than in the virtual world and that, for example, return to pre-COVID-19 levels of telephone or video consultations, if accompanied by a commensurate increase in in-person consultation, would be a positive development." (ID 910, Ireland)

\section{Subtheme 2.3. Remuneration issues}

- "Prior to COVID-19, video and telephone consultations [were] not remunerated by Medicare. Technology was available, but was paid by the user/patient, prior to COVID-19. The telehealth subsidy in Australia is scheduled to end by March 2021, so we will return to dark ages again because patients might not want to pay for healthcare - especially telephone or video consultations, which are seen as low value by patients preferring face to face and hands-on medical care." (ID 1603, Australia)

- "If government withdraws fees for phone and video consultations then I would be less inclined to use them and more inclined to bring patients into office (sometimes needlessly)" (ID 1245, Canada) 


\section{THEME 3: CHALLENGES FOR HEALTH CARE SYSTEMS}

Subtheme 3.1. Organisational culture

- "Tradition: "we have to return to what we did before... it worked..." (ID 178, Chile)

- "People don't like changes, especially if they have to do something differently, or additionally" (ID 1096, Croatia)

- "People [will] forget easily how digital ways of working facilitated work during the pandemic and [will] easily return to the familiar normality which they knew before the pandemic" (ID 10, Finland)

Subtheme 3.2. Technical challenges

- "There is no resources (computers, telephones) for all health workers, therefore not everyone can work through digital techs and personal resources need to be used such as personal phones" (ID 274, Chile)

- "EHR and network problems - overloaded, not prepared for massive digital use of systems, working very slowly. Still waiting for the solution (new server, better internet connection, etc)" (ID 55, Portugal)

- "Lack of efficient digital equipment and software able to communicate with each other, because up to now it means opening many windows over and over again" (ID 199, Chile)

- "No integration between systems and some of them do the same thing instead of complementing each other" (ID7, Portugal)

\section{Subtheme 3.3. Implementation issues}

- "The technologies were fine, but my health system did a terrible job implementing the changes. They were slow to find video services. They changed the type of video service three times." (ID 566, USA)

\section{Subtheme 3.4. Financial issues}

- "They furloughed so many staff personnel that physicians were expected to do digital appts with no staff support. There was no staff to answer phones except two days a week for two weeks. It was a mess.” (ID 566, USA)

- "Public organizations inability to see investments - only costs. Conservative culture within the organization." (ID 1414, Sweden)

- "Lack of financial investment from governments in such technologies; Patients emotional need to get in touch with medical/other health care workers staff; Specially in Brazil, it is possible that our Medical Council pressure up against telemedicine consultations because it is an issue that before the COVID19 pandemic situation they have never been sympathetic with." (ID 65, Brazil)

- $\quad$ "Short-sightedness of managers, Unequal distribution of resources, inbred inertia of large systems which resist change" (ID 11, Israel)

Subtheme 3.5. Legal and regulatory weaknesses

- "Current legislation limits teleconsultations" (ID 860, Chile)

- "Regulatory barriers across state and geographic lines - overly strict (...) regulations - prevent right care in right venue direction" (ID 1378, USA)

- "In my opinion, the main problem lies in the fact that, according to the Italian code of medical ethics, the medical examination can only take place in presence. This limit has significant repercussions on the lawfulness of the prescription and certification carried out during a video consultation." (ID 175, Italy)

\section{Healthcare providers}

The lack of formal training, guidance, and inadequate technical support were notable challenges. During the abrupt shift to virtual care, GPs reportedly had to set up, learn how to use, and troubleshoot new systems. 
These efforts were seldom well-organised or coordinated and often resulted in considerable transitional difficulties. Another major drawback was the higher workload and burnout. Some participants reported that they found virtual consultations to be more taxing and time-consuming to perform, which was often burdened with increased administrative tasks. As described above, the frequency and ease with which GPs could be contacted by patients often blurred the boundaries between their work and personal lives, further contributing to an increased workload. The increase in the number of patients observed daily has also resulted in less engagement with individual patients and decreased overall work satisfaction. GPs also reported inadequate remuneration as a considerable barrier to the continued use of virtual care. Whilst payment structures differed across countries, our respondents expressed that payment for remote consultation which is commensurate to in-person consultations, is critical to incentivising greater acceptance and mainstream use. As many of the remote consultation payment schemes were introduced during the initial months of COVID-19 pandemic as emergency measures, some GPs were doubtful that these efforts would be sustained over time.

\section{Health systems}

Participants mentioned organisational culture as an impediment against using virtual care. Overcoming substantial institutional inertia, the lack of pre-existing teleworking culture, the urgency to shift from familiar systems to completely new ones, and challenging the prevailing mentality of 'this was always how things were done', were commonly reported organisational barriers. Participants also reported technical issues with the digital systems used, including inadequate equipment and infrastructure, and poor systems interoperability. In particular, the lack of interoperability between several digital systems, such as appointment schedulers, electronic health records, electronic prescriptions, and epidemiological surveillance systems, was perceived as a major challenge. GPs also described implementation issues related to finances, including a general lack of investment and, more specifically, lack of funding allocated to human resources and supporting the costs of systems set-up and maintenance. Respondents noted that virtual care delivery did not appear to be in the list of priorities of many healthcare systems or policymakers. Finally, GPs identified a range of legal and regulatory weaknesses, highlighting that existing legislation is no longer fit for purpose, nor meets the evolving needs resultant from the pandemic.

265

266

Figure 2: Conceptual map of main challenges identified

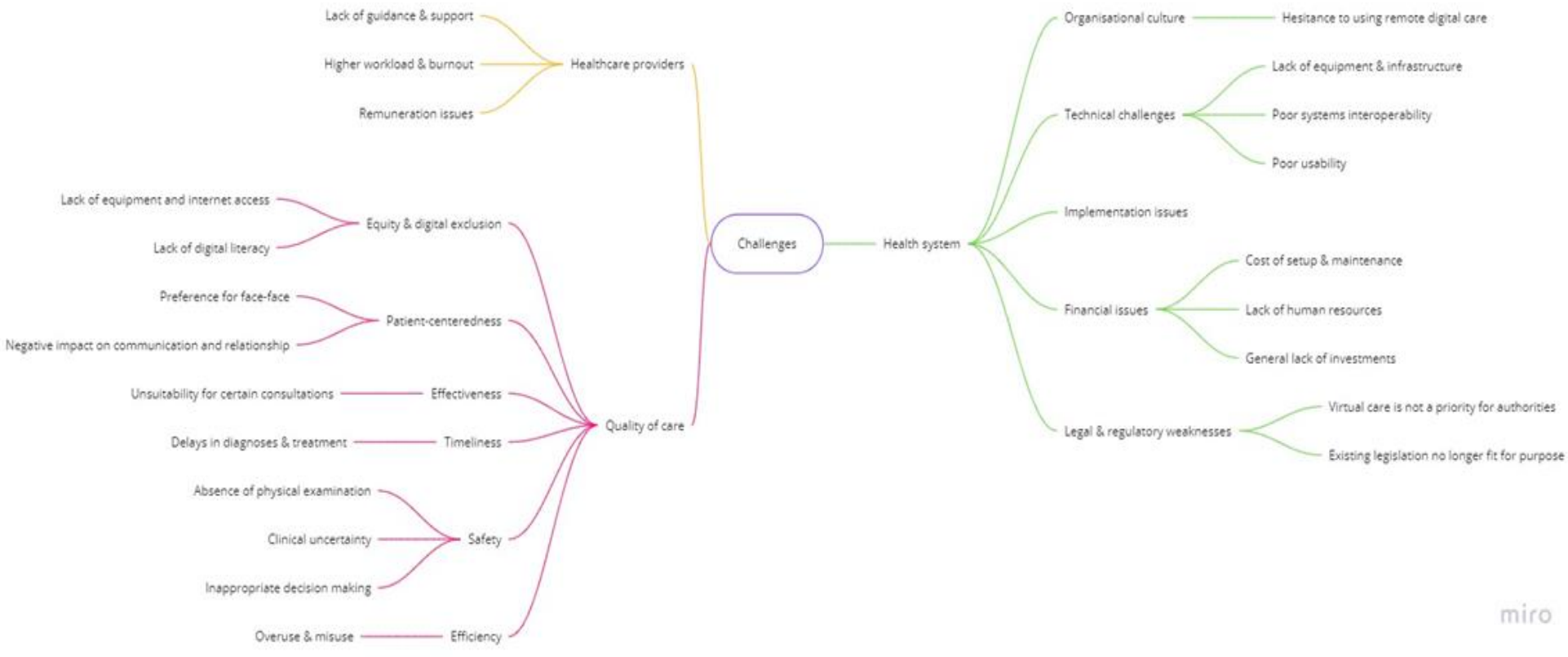




\section{Discussion}

Benefits for quality of care were identified for the six dimensions of quality of care, including safety (reduction of exposure risks for COVID-19 transmission); effectiveness and equity (ensuring access and continuity of care to those who need it, including those who had previously limitations to access face-toface care); efficiency (remote triage), improved timeliness and patient-centredness (convenience, communication, and patient empowerment). Benefits for healthcare providers included a greater work flexibility and more control over schedule. Benefits for healthcare systems included a transformative effect, hastening the digital transformation (increasing awareness, trust, adoption, skills, and technical capacity) and driving changes in legal and regulatory frameworks.

Important challenges have also been noted for six domain of quality of care, including patient-centredness (patients' preference for face-to-face care, potential negative impact on communication), equity (lack of equipment, internet access and digital skills of some patient groups), safety (clinical uncertainty and potentially inappropriate decision making), timeliness (delays in diagnosis and treatment), effectiveness healthcare providers included the lack of guidance and support, higher workload, and remuneration issues. From the health systems' perspective, the long-established organisational culture, technological challenges, implementation and financial issues, and lack of accompanying supportive policies and regulatory legislation, were challenges identified by participants.

\section{Strengths and limitations}

This is the first international study to explore GPs' perceptions on the main benefits and challenges of using virtual consultations in primary care. Participants took part from 20 countries worldwide, with diverse health care systems and levels of healthcare spending. The sample size was large, with participants varying in age, clinical experience, and type of primary care setting (urban, rural, or mixed). This study employed a methodologically rigorous approach, leveraging qualitative methods to capture rich, descriptive data on individual perceptions, attitudes, and behaviours [33,34], performed according to the Consolidated Criteria for Reporting Qualitative Studies criteria [35]. Finally, the main benefits and challenges were mapped against a widely recognised framework of Quality of Care [10], whenever possible. Finally, a set of recommendations was developed based on the main findings, to support providers and healthcare organisations translate the lessons learned into practice improvements.

The results must be interpreted considering some limitations. Our findings are also impacted by common limitations of survey research, including self-reported answers and self-selection sampling methods. Only GPs were included in this study; future research should focus on the inclusion of other healthcare professionals, and especially patients. The themes identified as part of this analysis may not be equally relevant for each individual country. Further qualitative content analysis could provide novel insights on their relative importance for individual countries. 
Remote primary care is widely recognised as an promising solution to ensuring both patient and provider safety by preventing direct physical contact, hence reducing morbidity and mortality during the COVID-19 pandemic [16,36,37]. However, important safety concerns also manifested, predominantly concerning diagnostic uncertainty. In this context, previous literature also report GPs' concerns about clinical risk [38] and the need to establish escalation protocols to support clinicians decide when a transition to urgent inperson follow-up care, or even to emergency services, is required [37].

Our results underline that remote digital tools may be an effective way of delivering primary care. In line with these findings, a Cochrane systematic review (2015) demonstrated the use of telemedicine strategies to be associated with increased access to care and improved clinical outcomes in single chronic diseases, particularly in type 2 diabetes [39]. However, the interventions were heterogenous and the external generalisability of these findings remain unclear. Future research will be needed to address questions such as for which patients, and for which conditions, do virtual care tools actually improve effectiveness.

Our participants highlighted that virtual care, particularly through remote triage, can reduce the number of unnecessary visits and thus have a positive impact on efficiency (i.e., minimising waste, including from an economic perspective). In fact, few telehealth evaluations have examined the association between outcomes and costs of virtual care. While some reviews have found that virtual care can decrease the use of acute hospital services [40-42], there is less evidence in the primary care context [43]. On the other hand, our participants raised concerns about potential overuse and misuse by patients, which could negatively impact the effectiveness of remote care. In a recent study in Canada (2020) evaluating the uptake of a platform for virtual visits in primary care, Stamenova et al. observed that many virtual visits appeared to replace faceto-face visits, yet patients did not overwhelm physicians with requests [44].

In what concerns timeliness of care, participants identified both potential advantages and disadvantages. Remote primary care has the potential to offer convenient access to a primary care provider without needing to take time-off work, arrange transportation, and spend time waiting for face-to-face visits. Participants were also concerned that barriers to the use of technology and difficulties inherent to a new mode of care delivery, could result in delays in diagnosis and treatment. There is sparse evidence on the subject. However, a recent study examined patient-initiated primary care visits in the Kaiser Permanente Northern California (a system with over four million members) and concluded that, on average, telephone visits were scheduled 50\% sooner than office visits [45]. These findings have profound implications, given that timeliness of care is associated with improved health outcomes. [46].

Remote care has been promised to reduce inequities in access to care for decades, particularly in rural and geographically remote areas [47-49]. In line with previous literature, our results demonstrate the ability of remote care to overcome barriers for those who have physical limitations to attend a face-to-face meeting, but also highlight their potential to entrench existing inequities in access to care [50,51]. Published evidence shows that the transition to virtual primary care did not unfold in the same manner across communities [52]. Proactive efforts are therefore needed to identify and address both patient and provider-related digital barriers so as to avoid that the widespread implementation of virtual care in a manner which reinforces disparities in health access amongst already underserved and excluded groups [52]. 
acknowledge that it can have both negative and positive effects on communication. Another important challenge is patient preference for face-to-face visits. Preference theory suggests that patients would prefer a virtual consultation if they perceive its benefits as outweighing its burdens [53]. Multiple factors may influence patients' preference, including the situation of care (i.e., patient's perception of their clinical status, treatment requirements, and care pathway), the expectations of care, the demand of care (e.g., social situation, consequences of choice), the capacity to allocate resources (e.g., patient's ability to allocate financial, infrastructural, social and healthcare resources) [54] and patients digital health literacy [55,56]. These factors may combine or compete and may be dynamic throughout the patient's journey.

The pandemic has a transformational impact in hastening the digital transformation, and in particular increasing awareness, trust, and adoption of remote care [16,57,58]. Challenges such as the lack of support, burnout, and remuneration issues, are balanced by a few benefits (i.e., remote working and a better control over schedule). However, to fully embrace the benefits postulated (e.g., greater flexibility to work remotely from home), healthcare systems must explore and implement the enablers required. Potential enablers include single sign-on, sharing of records across systems, electronic generation of form, and the creation of workspaces and secure interfaces without the need for laptops and VPNs [59]. While the rapid implementation of virtual consulting tools provides the ability to work more flexibly and from different locations, primary care leaders need to be supported and learn how to build effective teams via novel approaches [60].

At the health systems level, previous studies have indicated that implementation barriers largely depended on accreditation, payment systems, and insurance [61]. Prioritisation of financial investments into relevant infrastructure, greater emphasis on training and guidance for healthcare providers, and updates to the corresponding legal and regulatory frameworks supporting their use, are equally required. Overcoming institutional inertia is likely to be more feasible post-COVID-19, given that clinical culture is expected to have evolved substantially after a year of daily use of remote care delivery. In addition, as many of the existing guidance and policies on primary remote care were drafted and implemented during the emergency phase, the experience attained, and evidence collected offers an opportunity to refine, optimise, and update the relevant accompanying legal and regulatory frameworks.

\section{Implications for research and policy}

Based on our findings, we propose a framework of recommendations for the implementation of virtual care in the primary care setting (Figure 3). The framework consists of suggested action points across five specific domains for high-quality implementation of virtual care, including contextual considerations, technology infrastructure, awareness and experience, safety and risk management, and strategic planning \& supporting policies (Figure 3). As part of this framework, we emphasise the importance of continuously monitoring quality across these five areas, which must be collected both through patient \& provider feedback, but also using data-driven approaches to systematically evaluate the impact on equity (e.g., usage patterns and patients characteristics, patient preferences, and offer of alternative modes of consultation), effectiveness (e.g., longitudinal monitoring of impact on health outcomes, as well as of the technological, legal and regulatory infrastructure required to deliver effective care), and safety (e.g., longitudinal monitoring of safety indicators, including cybersecurity); efficiency (e.g., cost-effectiveness analysis) and timeliness (e.g., time-to-diagnosis and time-to-treatment). Decisions to adopt remote care services should 402 
medRxiv preprint doi: https://doi.org/10.1101/2021.07.28.21261021; this version posted July 29, 2021. The copyright holder for this preprint (which was not certified by peer review) is the author/funder, who has granted medRxiv a license to display the preprint in perpetuity.

It is made available under a CC-BY 4.0 International license .

It is important to note that the technological and regulatory landscape is dynamic, as are patients' preferences. Therefore, virtual care platforms must be secure and reliable, but also flexible enough to accommodate evolving regulatory, professional, and health-care organisations' requirements [62] - and processes must ensure that patients are presented with alternative options during their journey.

Figure 3: A framework for implementation of virtual consultations in the primary care setting
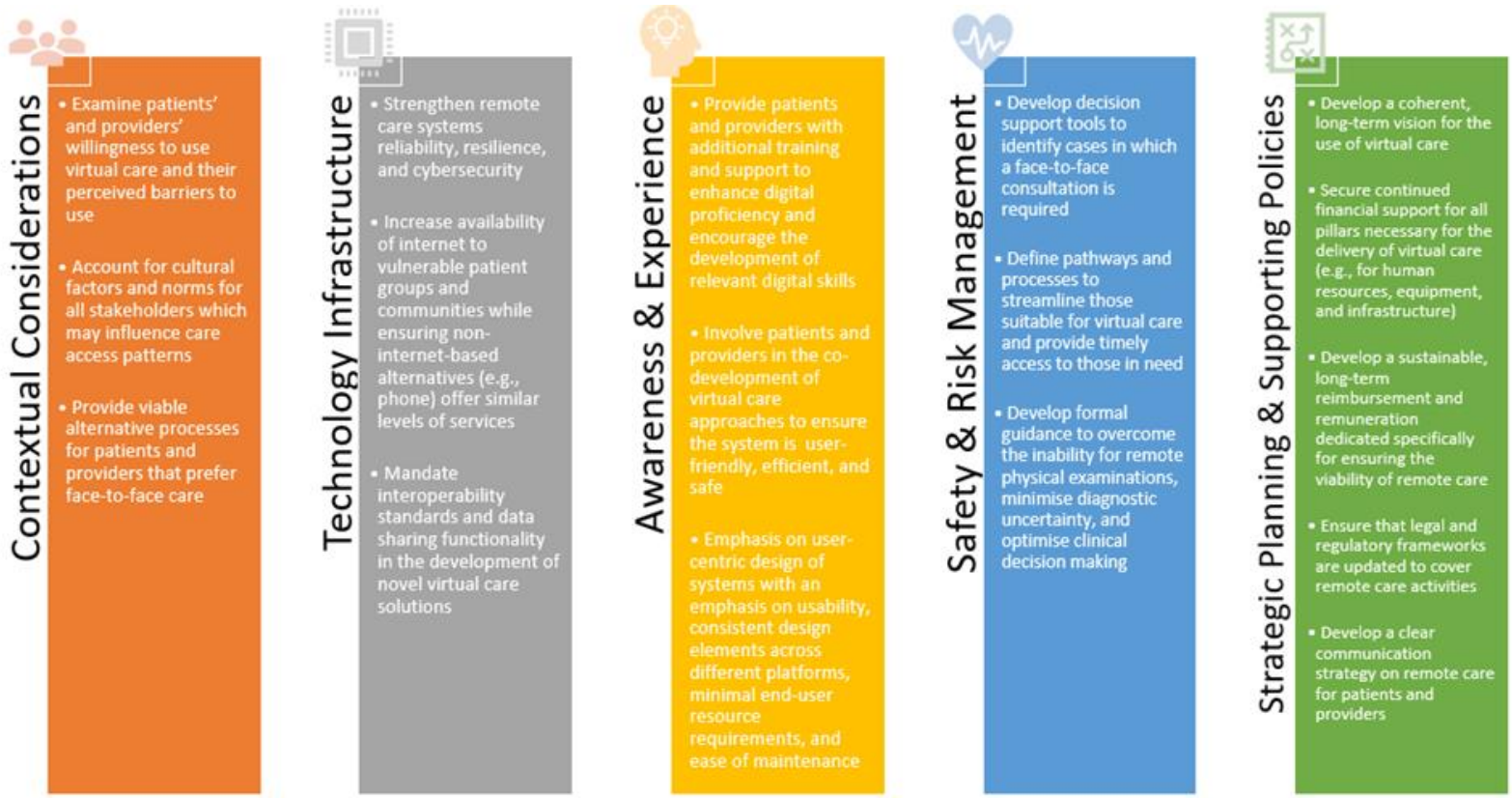

Monitoring \& Evaluation

To systematically capture information and evaluate the impact on the five areas identified

Ta consider the impact on the six domains of quality of care (patient-centredness, effectiveness, efficiency, safety, timeliness and equity)

Capitalize on a variety of data sources, including patient and provider feedback, but also using data-driven approaches

Use evidence to inform decisions to adopt remote care services

\section{Conclusion}

At the frontlines of care delivery, GPs can provide valuable insights into the use of remote care - what worked well, how, and why. Experience gained during the emergency phase of the COVID-19 pandemic can be used to inform the stable adoption of virtual care solutions, the co-designing of processes and platforms that are technologically robust, and supported by a strategic long-term plan. The development of such platforms should consider pre-existing health inequities and contextual considerations, and deliberately implement strategies to minimise digital exclusion and optimise patients' experience. Finally, primary care health systems need to rigorously evaluate the ongoing impact on virtual primary care both during and after the pandemic, and incorporate the lessons learned into legal and regulatory frameworks that support its long-term, sustainable use.

\section{Funding}

This research was supported through the Imperial College National Institute for Health Research (NIHR) Patient Safety Translational Research Centre (PSTRC) and the European General Practice Research Network (EGPRN). The funders/sponsors have had no role in development and drafting of this manuscript. 
medRxiv preprint doi: https://doi.org/10.1101/2021.07.28.21261021; this version posted July 29, 2021. The copyright holder for this preprint (which was not certified by peer review) is the author/funder, who has granted medRxiv a license to display the preprint in perpetuity.

It is made available under a CC-BY 4.0 International license .

\section{References}

1 Pearl R. Kaiser Permanente Northern California: Current Experiences With Internet, Mobile, And Video Technologies. https://doi.org/101377/hlthaff20131005 2017;33:251-7. doi:10.1377/HLTHAFF.2013.1005

$2 \quad$ Van Galen LS, Car J. Telephone consultations. BMJ 2018;360. doi:10.1136/bmj.k1047

3 McGrail KM, Ahuja MA, Leaver CA. Virtual Visits and Patient-Centered Care: Results of a Patient Survey and Observational Study. J Med Internet Res 2017;19. doi:10.2196/JMIR.7374

4 Fatehi F, Menon A, Bird D. Diabetes Care in the Digital Era: a Synoptic Overview. Curr Diabetes Reports 2018187 2018;18:1-8. doi:10.1007/S11892-018-1013-5

5 Greenhalgh T, Vijayaraghavan S, Wherton J, et al. Virtual online consultations: advantages and limitations (VOCAL) study. BMJ Open 2016;6:e009388. doi:10.1136/BMJOPEN-2015-009388

6 NHS England and British Medical Association. Investment and evolution: a five-year framework for GP contract reform to implement the NHS long term plan. 2019.

7 Digital Health and Care Scotland. Scotland's Digital Health and Care Strategy: enabling, connecting and empowering. 2018;:20.https://www.gov.scot/publications/scotlands-digital-healthcare-strategy-enabling-connecting-empowering/ (accessed 13 Jul 2021).

8 US Department of Veterans Affairs. Department of Veterans Affairs FY 2018-2024 Strategic Plan. https://www.va.gov/oei/docs/VA2018-2024strategicPlan.pdf (accessed 13 Jul 2021).

9 National Digital Health Strategy and Framework for Action | Australian Digital Health Agency. https://www.digitalhealth.gov.au/about-us/national-digital-health-strategy-and-framework-foraction (accessed $13 \mathrm{Jul} 2021$ ).

10 of Medicine I. Crossing the Quality Chasm: A New Health System for the 21 st Century. Washington, DC: : The National Academies Press 2001. doi:10.17226/10027

11 Polinski JM, Barker T, Gagliano N, et al. Patients' Satisfaction with and Preference for Telehealth Visits. J Gen Intern Med 2016;31:269-75. doi:10.1007/s11606-015-3489-x

12 Thiyagarajan A, Grant C, Griffiths F, et al. Exploring patients' and clinicians' experiences of video consultations in primary care: A systematic scoping review. BJGP Open 2020;4. doi:10.3399/bjgpopen20X101020

13 Jong M, Mendez I, Jong R. Enhancing access to care in northern rural communities via telehealth. Int J Circumpolar Health 2019;78. doi:10.1080/22423982.2018.1554174

14 Bradford NK, Caffery LJ, Smith AC. Correction: Telehealth services in rural and remote Australia: A systematic review of models of care and factors influencing success and sustainability [Rural and Remote Health, 16, (2016) 4268]. Rural Remote Health. 2016;16. doi:10.22605/RRH4268

15 Li JPO, Thomas AAP, Kilduff CLS, et al. Safety of video-based telemedicine compared to inperson triage in emergency ophthalmology during COVID-19. EClinicalMedicine 2021;34. doi:10.1016/j.eclinm.2021.100818

16 Monaghesh E, Hajizadeh A. The role of telehealth during COVID-19 outbreak: A systematic review based on current evidence. BMC Public Health. 2020;20:1-9. doi:10.1186/s12889-02009301-4

17 Lin $\mathrm{CH}$, Tseng WP, Wu JL, et al. A double triage and telemedicine protocol to optimize infection control in an emergency department in Taiwan during the COVID-19 pandemic: Retrospective feasibility study. J Med Internet Res 2020;22. doi:10.2196/20586

18 Roberts A, Garrett L, Godden DJ. Can telehealth deliver for rural Scotland? lessons from the argyll \& bute telehealth programme. Scott Med J 2012;57:33-7. doi:10.1258/smj.2011.011288

19 Terschüren C, Fendrich K, van den Berg N, et al. Implementing telemonitoring in the daily routine of a GP practice in a rural setting in northern Germany. J Telemed Telecare 2007;13:197-201. doi:10.1258/135763307780908003

20 Steventon A, Bardsley M, Billings J, et al. Effect of telehealth on use of secondary care and mortality: Findings from the Whole System Demonstrator cluster randomised trial. BMJ 2012;344. doi:10.1136/bmj.e3874 
medRxiv preprint doi: https://doi.org/10.1101/2021.07.28.21261021; this version posted July 29, 2021. The copyright holder for this preprint (which was not certified by peer review) is the author/funder, who has granted medRxiv a license to display the preprint in perpetuity.

It is made available under a CC-BY 4.0 International license .

Affinito L, Fontanella A, Montano N, et al. How physicians can empower patients with digital tools: A joint study of the Italian Scientific Society of Internal Medicine (FADOI) and the European Federation of Internal Medicine (EFIM). J Public Heal Published Online First: 2020. doi:10.1007/s10389-020-01370-4

22 McGloin H, O'Connell D, Glacken M, et al. Patient empowerment using electronic telemonitoring with telephone support in the transition to insulin therapy in adults with type 2 diabetes: Observational, pre-post, mixed methods study. J Med Internet Res 2020;22. doi:10.2196/16161

23 Bower P, Cartwright M, Hirani SP, et al. A comprehensive evaluation of the impact of telemonitoring in patients with long-term conditions and social care needs: protocol for the whole systems demonstrator cluster randomised trial. BMC Heal Serv Res 2011111 2011;11:1-12. doi:10.1186/1472-6963-11-184

24 European Commission. Market study on telemedecine. Eur Comm 2018;:132.http://europa.eu (accessed 13 Jul 2021).

25 Majeed A, Maile EJ, Bindman AB. The primary care response to COVID-19 in England's National Health Service. J. R. Soc. Med. 2020;113:208-10. doi:10.1177/0141076820931452

26 Tsopra R, Frappe P, Streit S, et al. Reorganisation of GP surgeries during the COVID-19 outbreak: analysis of guidelines from 15 countries. BMC Fam Pract 2021;22:1-16. doi:10.1186/s12875-02101413-z

27 Andrea Downey. Coronavirus: GPs urged to go digital to prevent spread of COVID-19. 2020.https://www.digitalhealth.net/2020/03/gps-urged-to-go-digital-to-prevent-spread-ofcoronavirus/ (accessed 13 Jul 2021).

28 Neves A, Lygidakis H, Fontana G. The technology legacy of COVID-19 in primary care. bjgplife.com Published Online First: 2020.https://bjgplife.com/2020/04/15/the-technology-legacyof-covid-19-in-primary-care/ (accessed 24 Nov 2020).

29 Shaghaghi A, Bhopal RS, Sheikh A. Approaches to Recruiting 'Hard-To-Reach' Populations into Re-search: A Review of the Literature. Heal Promot Perspect 2011;1:86-94. doi:10.5681/hpp.2011.009

30 Atkinson R, Flint J. Social Research Update 33: Accessing Hidden and Hard-to-Reach Populations. Soc Res Updat 2001;Summer:3.https://sru.soc.surrey.ac.uk/SRU33.html (accessed 13 Jul 2021).

31 Avery L, Rotondi N, McKnight C, et al. Unweighted regression models perform better than weighted regression techniques for respondent-driven sampling data: Results from a simulation study. BMC Med Res Methodol 2019;19:1-13. doi:10.1186/s12874-019-0842-5

32 About Miro| Meet the team | Our mission. https://miro.com/about/ (accessed 22 Jul 2021).

33 Suter W. Introduction to Educational Research: A Critical Thinking Approach. Introd to Educ Res A Crit Think Approach Published Online First: 23 December 2014. doi:10.4135/9781483384443

34 Al-Busaidi ZQ. Qualitative research and its uses in health care. Sultan Qaboos Univ. Med. J. 2008;8:11-9.

35 Booth A, Hannes K, Harden A, et al. COREQ (Consolidated Criteria for Reporting Qualitative Studies). Guidel Report Heal Res A User's Man 2014;:214-26. doi:10.1002/9781118715598.CH21

36 Majeed A, Molokhia M, Pankhania B, et al. Protecting the health of doctors during the COVID-19 pandemic. Br J Gen Pract 2020;70:268. doi:10.3399/BJGP20X709925

37 Sikka N, Willis J, Fitall E, et al. Telehealth and Patient Safety During the COVID-19 Response | PSNet. 2020.https://psnet.ahrq.gov/perspective/telehealth-and-patient-safety-during-covid-19response (accessed $13 \mathrm{Jul} 2021$ ).

38 Murphy M, Scott LJ, Salisbury C, et al. Implementation of remote consulting in UK primary care following the COVID-19 pandemic: A mixed-methods longitudinal study. Br J Gen Pract 2021;71:E166-77. doi:10.3399/BJGP.2020.0948

39 Flodgren G, Rachas A, Farmer AJ, et al. Interactive telemedicine: Effects on professional practice and health care outcomes. Cochrane Database Syst. Rev. 2015;2015. 
medRxiv preprint doi: https://doi.org/10.1101/2021.07.28.21261021; this version posted July 29, 2021. The copyright holder for this preprint (which was not certified by peer review) is the author/funder, who has granted medRxiv a license to display the preprint in perpetuity.

It is made available under a CC-BY 4.0 International license.

doi:10.1002/14651858.CD002098.pub2

40 Inglis SC, Clark RA, McAlister FA, et al. Structured telephone support or telemonitoring programmes for patients with chronic heart failure. In: Cochrane Database of Systematic Reviews. Cochrane Database Syst Rev 2010. doi:10.1002/14651858.cd007228.pub2

41 Henderson C, Knapp M, Fernández JL, et al. Cost effectiveness of telehealth for patients with long term conditions (Whole Systems Demonstrator telehealth questionnaire study): Nested economic evaluation in a pragmatic, cluster randomised controlled trial. BMJ 2013;346. doi:10.1136/bmj.f1035

42 Polisena J, Tran K, Cimon K, et al. Home telehealth for chronic obstructive pulmonary disease: A systematic review and meta-analysis. J Telemed Telecare 2010;16:120-7. doi:10.1258/jtt.2009.090812

43 Polisena J, Tran K, Cimon K, et al. Home telehealth for diabetes management: A systematic review and meta-analysis. Diabetes, Obes. Metab. 2009;11:913-30. doi:10.1111/j.14631326.2009.01057.x

44 Stamenova V, Agarwal P, Kelley L, et al. Uptake and patient and provider communication modality preferences of virtual visits in primary care: a retrospective cohort study in Canada. $B M J$ Open 2020;10:e037064. doi:10.1136/bmjopen-2020-037064

45 Graetz I, Huang J, Muelly E, et al. Patient choice of telemedicine increases timeliness of primary care visits. AMIA Annu Symp Proc 2020;2020:502./pmc/articles/PMC8075419/ (accessed 13 Jul 2021).

46 Ray KN, Chari A V., Engberg J, et al. Disparities in Time Spent Seeking Medical Care in the United States. JAMA Intern Med 2015;175:1983-6. doi:10.1001/JAMAINTERNMED.2015.4468

47 Howland M, Tennant M, Bowen DJ, et al. Psychiatrist and Psychologist Experiences with Telehealth and Remote Collaborative Care in Primary Care: A Qualitative Study. J Rural Heal Published Online First: 2020. doi:10.1111/JRH.12523

48 Fabian Buddy Pascoal, Nadya Vera Simanjuntak and WA. The Rise of Telemedicine in Indonesia. Dentons HPRP. 2021.https://www.dentons.com/en/insights/articles/2020/july/20/therise-of-telemedicine-in-indonesia (accessed 13 Jul 2021).

49 Perri MG, Shankar MN, Daniels MJ, et al. Effect of Telehealth Extended Care for Maintenance of Weight Loss in Rural US Communities: A Randomized Clinical Trial. JAMA Netw open 2020;3:e206764. doi:10.1001/jamanetworkopen.2020.6764

50 Greenhalgh T, Shaw S, Wherton J, et al. Real-world implementation of video outpatient consultations at macro, meso, and micro levels: Mixed-method study. J Med Internet Res 2018;20. doi:10.2196/jmir.9897

51 Brant $\mathrm{H}$, Atherton $\mathrm{H}$, Ziebland $\mathrm{S}$, et al. Using alternatives to face-to-face consultations: A survey of prevalence and attitudes in general practice. Br J Gen Pract 2016;66:e460-6. doi:10.3399/bjgp16X685597

52 Chang JE, Lai AY, Gupta A, et al. Rapid Transition to Telehealth and the Digital Divide: Implications for Primary Care Access and Equity in a Post-COVID Era. Milbank Q 2021;99:340 68. doi:10.1111/1468-0009.12509

53 Hausman DM. EconPapers: Preference, Value, Choice, and Welfare. Cambridge Univ. Press. 2012.https://econpapers.repec.org/bookchap/cupcbooks/9781107695122.htm (accessed $13 \mathrm{Jul}$ 2021).

54 Gilbert AW, Jones J, Stokes M, et al. Factors that influence patient preferences for virtual consultations in an orthopaedic rehabilitation setting: A qualitative study. BMJ Open 2021;11:e041038. doi:10.1136/bmjopen-2020-041038

55 Levy H, Janke AT, Langa KM. Health Literacy and the Digital Divide Among Older Americans. $J$ Gen Intern Med 2015;30:284. doi:10.1007/S11606-014-3069-5

56 Zide M, Caswell K, Peterson E, et al. Consumers' Patient Portal Preferences and Health Literacy: A Survey Using Crowdsourcing. JMIR Res Protoc 2016;5:e104. doi:10.2196/resprot.5122

57 Ahmed S, Sanghvi K, Yeo D. Telemedicine takes centre stage during COVID-19 pandemic. BMJ 
medRxiv preprint doi: https://doi.org/10.1101/2021.07.28.21261021; this version posted July 29, 2021. The copyright holder for this preprint (which was not certified by peer review) is the author/funder, who has granted medRxiv a license to display the preprint in perpetuity.

It is made available under a CC-BY 4.0 International license.

Innov. 2020;6:252-4. doi:10.1136/bmjinnov-2020-000440

58 Webster P. Virtual health care in the era of COVID-19. Lancet (London, England). 2020;395:1180-1. doi:10.1016/S0140-6736(20)30818-7

59 COVID-19: video consultations and homeworking. https://www.bma.org.uk/advice-andsupport/covid-19/adapting-to-covid/covid-19-video-consultations-and-homeworking (accessed 13 Jul 2021).

60 Baird B, Beech J, Chauhan K, et al. Remote working toolkit for general practices and primary care networks $\mid$ The King's Fund. Kings Fund. 2021.https://www.kingsfund.org.uk/publications/remote-working-toolkit-general-practices-pcns (accessed 13 Jul 2021).

61 Hollander JE, Carr BG. Virtually Perfect? Telemedicine for Covid-19. N Engl J Med 2020;382:1679-81. doi:10.1056/nejmp2003539

62 Blandford A, Wesson J, Amalberti R, et al. Opportunities and challenges for telehealth within, and beyond, a pandemic. Lancet Glob. Heal. 2020;8:e1364-5. doi:10.1016/S2214-109X(20)30362-4 

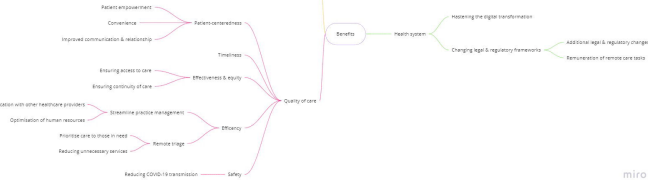
Lovof puosonos severt

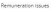

Haveress prende?

Heper werlisod nod sement

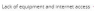

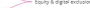

Protementisersestact -

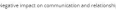

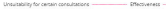

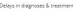

Thes ras

sus of of phoce sani-sol

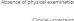

In

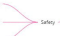

Orgascatons owheve

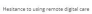

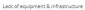

Tachesel thilevas

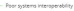

Peerivabla? Heast b,ase

- inpleversenionicios

Cooc of seow to mor monere

Prenod act Lodothumen tescuros:

Embral ladi of inverneno

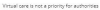

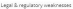




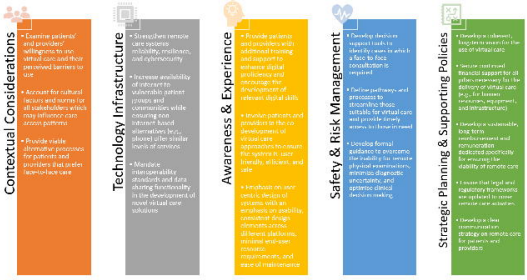

Monitoring \& Evaluation

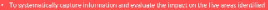

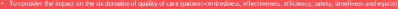

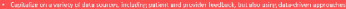

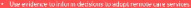

